J Pain Symptom Manage. 2019 July ; 58(1): 80-85. doi:10.1016/j.jpainsymman.2019.04.012.

\title{
Prevalence, Severity, and Correlates of Symptoms of Anxiety and Depression at the Very End of Life
}

\author{
Elissa Kozlov, PhD, Veerawat Phongtankuel, MD, Holly Prigerson, PhD, Ronald Adelman, \\ MD, Ariel Shalev, BA, Sara Czaja, PhD, Ritchell Dignam, MD, Rosemary Baughn, MSN, M. \\ Carrington Reid, MD, PhD \\ Weill Cornell Medicine (E.K., V.P., H.P., R.A., A.S., S.C., M.C.R.), Division of Geriatric and \\ Palliative Medicine, New York, New York; and Visiting Nurse Service of New York (R.D., R.B.), \\ New York, New York, USA
}

\begin{abstract}
Context.-Rates of psychological symptoms for patients with serious illness are high, but there has been limited research investigating psychological symptoms at the very end of life (EOL).

Objectives.-To better understand the prevalence, severity, and correlates of psychological distress at the very EOL.

Methods.-This retrospective cross-sectional study utilized caregiver proxy interviews. Caregivers were contacted after their care recipient recently died while receiving home hospice care and invited to participate in a brief interview with a trained research assistant. Patient, caregiver, and hospice utilization data were also abstracted from electronic medical records, and caregiver burden scores were assessed via interview.
\end{abstract}

Results.-A total of 351 caregivers were included in the study. According to caregivers, $46.4 \%$ of patients had moderate-to-severe anxiety, as assessed with a score of $\_4$ on the Edmonton Symptom Assessment Scale and 43\% had moderate-to-severe symptoms (Edmonton Symptom Assessment Scale score 24) of depression in the last week of life. Symptoms of anxiety and depression were significantly associated with caregiver burden scores and inversely associated with patient age.

\begin{abstract}
Conclusion.-Psychological symptom management at the very EOL is essential to providing comprehensive hospice care. Our study revealed that nearly half of all home hospice patients experience moderate-to-severe symptoms of anxiety and/or depression in the last week of life. Future research is needed to improve psychological symptom management at the very EOL to improve the quality of life for both patients and their families.
\end{abstract}

\section{Keywords}

Anxiety; depression; hospice; psychological symptoms; end of life

Address correspondence to: Elissa Kozlov, PhD, Weill Cornell Medicine, Division of Geriatric and Palliative Medicine, 525 East 68 th Street, Box 39, 1404 Baker Pavilion, New York Presbyterian Hospital, New York City, NY 10065, USA. elk2020@ med.cornell.edu. 


\section{Introduction}

Good symptom management, which includes effectively managing psychological symptoms, is essential for quality end-of-life (EOL) care. Rates of psychological symptoms for patients with serious illnesses are high, ${ }^{1-4}$ but there has been limited research on psychological symptoms at the very EOL. The few publications on this topic suggest that psychological symptom burden remains high up until death. ${ }^{5,6}$ One study found that the prevalence of depression symptoms was $38.6 \%$ and $43.5 \%$ for anxiety symptoms in patients with cancer during their last weeks of life. ${ }^{5}$ Another study investigating hospice patients found that substantial proportions of patients exhibited signs of sadness (50\%), worry (43\%), and nervousness (42\%), as perceived by hospice providers. ${ }^{6}$ Preliminary research on patients with cancer enrolled in hospice suggests that rates of anxiety and depression may be as range from $42 \%$ to $70 \%,{ }^{7-9}$ but more research is needed to better understand the prevalence, intensity, and correlates of psychological distress at the very EOL.

Assessing psychological symptoms at the very EOL is methodologically challenging. The "gold standard" method includes patient ratings, but many patients are unable to participate in clinical interviews in the last weeks of life. Using proxy assessors is often required and provides a relevant source of information, given that treatment decisions are frequently influenced by multiple stakeholders' (e.g., family, doctors, and home health providers) perception of the patient's symptom experience. ${ }^{6}$ Proxies, however, often have difficulty accurately assessing psychological symptoms. ${ }^{6}$ One study interviewed family members after the death of their loved one to inquire about symptoms the patient experienced in the last week of life. Twenty-eight percent of family members reported that their loved one was depressed, $31 \%$ endorsed that their loved one exhibited signs of anxiety, but only $35 \%$ reported that they spoke with the patient explicitly about the symptoms. It is unclear if proxy report of psychological symptoms are overestimates or underestimates of actual psychological symptom burden, ${ }^{10}$ but caregiver impressions of patient suffering, especially at the very EOL, are relevant given the large role caregivers often play in medical decisionmaking at EOL.

Given that 1.4 million patients ${ }^{11}$ use hospice services ${ }^{7}$ annually in the U.S., it is essential to better understand the prevalence and correlates of psychological symptoms in patients at the EOL to better serve our aging population. Effective symptom management is a primary goal of hospice care, but it remains unclear how often psychological symptoms are detected and/or treated. Accordingly, this study sought to determine the levels of psychological symptom distress as measured by the Edmonton Symptom Assessment Scale (ESAS), as perceived by caregivers, in home hospice patients in the last week of life. As a secondary objective, we sought to identify the correlates of symptoms of anxiety and depression. Identifying correlates of psychological symptom distress is important to determine to better understand and pre-emptively manage factors that increase risk for psychological symptom distress. 


\section{Methods}

\section{Study Design}

This secondary data analysis utilized data obtained from a retrospective cross-sectional study. The primary objective of the original study was to analyze correlates of patient symptom burden in the home hospice setting using caregiver proxy interviews. The study was approved by the Institutional Review Boards of Weill Cornell Medicine and the Visiting Nurse Service of New York.

The Visiting Nurse Service of New York Hospice and Palliative Care (VNSNYHPC) is a nonprofit hospice that serves over 1000 patients daily and delivers home hospice care to patients in the greater New York City area. VNSNYHPC provides home visits by an interdisciplinary team of physicians, nurses, social workers, and spiritual care counselors, as well as a medication kit for pain and symptom management and educational materials that describe available support services. A 24/7 phone service is staffed by a hospice on-call team, and a hospice practitioner may be dispatched to the home based on the needs of the patient and family.

\section{Participant Recruitment and Data Collection}

We enrolled caregivers whose care recipients recently died (two to three weeks ago) after receiving home hospice. Caregivers received a mailed letter introducing the study and informing them to expect a call in two weeks from a member of the research team. Eligible caregiver participants had to be 18 years or older, English speaking, and listed as a primary caregiver (e.g., family or friend) at the time the patient was admitted to the VNSNYHPC service.

A trained research assistant telephoned potential participants, described the study, and obtained verbal consent. A semistructured interview guide (described below) was administered during the phone call, and caregivers received a $\$ 25$ gift card for their participation. Data were entered into Research Electronic Data Capture (REDCap), a secure Web application for building and managing databases.

Patient, caregiver, and hospice utilization data were also abstracted from VNSNYHPC's electronic medical records on a weekly basis. This information included patient data (age, gender, hospice diagnosis) and the caregiver contact information (name, address, phone number).

\section{Caregiver Questions}

Dependent Variable.-Caregivers served as proxy respondents to estimate patient symptom burden during the last week of patients who received hospice care. Symptoms were assessed using the ESAS, a multi-symptom assessment scale frequently used in studies of patients with terminal illnesses, patients at the EOL, and with proxy reports. ${ }^{12-14}$ Caregivers were asked to recall symptom burden across the nine domains present in the ESAS, including anxiety and depression, during the patient's last week on hospice. 
Correlates.-The following variables were collected during phone interviews with caregiver participants: relationship with the patient, caregiver burden score, and level of comfort managing patient's symptoms. Caregiver burden was measured using the short version of the Burden Scale for Family Caregivers, an interviewer- or self-administered 10item scale with a range of $0-30$ with higher scores indicating more burden. ${ }^{15}$ Because there is no validated measure of caregiver comfort in managing patients' symptoms, we asked caregivers: "how would you rate your level of comfort managing (patient's name) symptoms during the last week on home hospice care?" Response rates ranged from 1 (very comfortable) to 5 (very uncomfortable). Caregiver satisfaction with care was assessed using the FAMCARE-17 scale. ${ }^{16}$ The FAMCARE is a 17 -item scale that measures the degree to which family members are satisfied with the health care received by both the patient and the family with respect to information giving, availability of care, psychological care, and physical patient care. It has been widely used in palliative care research, specifically in the palliative care setting and is administered via interview or self-report. Scores range from 17 to 68 , with lower scores representing higher satisfaction.

\section{Statistical Approach}

Descriptive statistics were used to determine rates of anxiety and depression symptoms. Depression and anxiety scores were trichotomized to represent mild, moderate, and severe symptoms based on the corresponding items on the ESAS. Scores of 0-3 were considered none to mild, 4-6 moderate, and 7 or higher (out of 10) severe symptoms. ${ }^{17}$ A series of bivariate correlations were conducted to determine associations between continuous variables (caregiver burden, satisfaction with hospice, age of patient, caregiver comfort with providing care) with the nontrichotomized ESAS. Analyses of variance were utilized to determine associations between categorical variables (caregiver relationship to patient, gender of caregiver, gender of patient, gender of caregiver, and cancer/noncancer diagnosis) and the continuous dependent variable (depression or anxiety item, measured 1-10, on the ESAS).

\section{Results \\ Sample Characteristics}

Of the 1848 caregivers called, 804 (44\%) never answered the phone, $653(35 \%)$ declined to participate, whereas 391 (21\%) met eligibility criteria and enrolled in the parent study. An additional $40(2 \%)$ caregivers were not eligible for the present study because their care recipient had a live discharge from hospice. The original study recruited a total of 391 caregiver participants. The full sample of caregiver proxies $(n=351)$ who had a patient die on hospice was used for analyses in the present study. Caregivers were primarily children of the deceased (60\%), spouses (17.7\%), and other relatives (14.8\%). Caregivers had a mean age of 59.7 years $(\mathrm{SD}=12.466)$ and were primarily female $(74.9 \%)$ and white $(47.9 \%)$. Patients had a mean age of 83.4 years $(\mathrm{SD}=14.1)$ and were primarily female $(66.1 \%)$ and white (55\%). 


\section{Prevalence Rates}

According to caregivers, $46.4 \%$ of patients had moderate-to-severe anxiety (a score of $\$ 4$ on the ESAS) symptoms in the last week of life (Table 1). For depressive symptoms, caregivers reported that $43 \%$ had moderate-to-severe symptoms of depression in the last week of life. See Table 1 for complete prevalence data.

\section{Associations}

Tables 2 and 3 present the bivariate correlation and analyses of variance results for the examined relationships between symptoms of depression, symptoms of anxiety, and relevant additional variables. There was no significant difference in perceived symptoms of depression or anxiety based on caregiver relationship, gender of caregiver, gender of patient, patient diagnosis (cancer or other), or caregiver comfort with providing care.

Symptoms of anxiety and depression were both significantly associated with caregiver burden scores (levels of perceived anxiety and depressive symptom distress were increased in those with higher caregiver burden scores) and patient age (younger patients had more perceived symptoms of depression and anxiety). Satisfaction with hospice, as assessed with the FAMCARE (where higher scores are associated with less satisfaction), was significantly associated with level of anxiety symptoms but not depression symptom score.

\section{Discussion}

This study contributes to the limited literature regarding the prevalence and severity of psychological symptoms at the very EOL and extends the prior literature by identifying correlates of psychological symptom burden at the EOL. Rates of moderate-to-severe symptoms of depression and anxiety, as perceived by caregivers, were $43 \%$ and $46 \%$, respectively. Psychological symptoms were significantly and positively associated with caregiver burden and inversely associated with patient age, and perceived severity of anxiety symptoms was associated with satisfaction with hospice care. Psychological symptoms in patients at the EOL are burdensome to both the patient and the caregiver, and attempts to both assess and treat psychological distress throughout the illness trajectory, including the last week of life, are clearly warranted.

Our study's prevalence rates are comparable to prior studies, which found rates of depression and anxiety symptoms that ranged from 38\% to 50\% among patients receiving hospice care. ${ }^{6}$ If nearly half of all patients receiving home hospice services experience moderate-to-severe levels of anxiety or depression symptomatology in the last week of life, then we need to improve the ways we address psychological distress at the EOL. Because hospice patients are likely receiving the highest levels of supportive care of patients dying in America, one can speculate that rates of psychological distress are even higher in patients dying without hospice services, for example, in the acute care setting.

Our results have several clinical practice implications. Providers caring for patients at the EOL need to pay attention to psychological symptoms in addition to the more often focused on physical symptoms (e.g., pain, dyspnea, and nausea). Further research is needed to establish better methodology and provider assessment practices for psychological 
assessment in patients at the very EOL. Although full diagnostic interviews with patients are not a viable option, earlier assessment of psychological distress using gold standard screeners, such as the Patient Health Questionnaire- $9^{18}$ and the generalized anxiety disorder- $7,{ }^{19}$ would be useful starting points to help manage psychological distress at EOL. Future research and quality improvement programs could aim to not only improve provider protocols to screen for psychological symptoms but also to train caregivers to recognize psychological distress in patients at EOL. Understanding the nonverbal manifestations of depression and anxiety in this target population could inform future educational programs to help increase provider and caregiver awareness of psychological distress in patients at the EOL. Research in other areas, such as intubated patients in the ICU and dementia patients in nursing homes, has attempted to detect anxiety and depression symptoms in nonverbal patients ${ }^{20-22}$ and may be applicable to home hospice patients.

This study determined that patient age is negatively and significantly correlated with increased severity of symptoms of anxiety and depression, as perceived by the caregiver. Given these results, it may be even more important for clinicians to be aware of psychological symptom burden in younger hospice patients. It may also be that younger patients are perceived by their caregivers as having more psychological distress because the caregivers themselves are more distressed at the impending loss of a younger person. Further research evaluating the role of patient age on psychological symptoms at the EOL is warranted given this preliminary finding.

This study also found that caregiver burden was significantly associated with patient symptoms of anxiety and depression. Other caregiving research has shown that patient mental health is a significant correlate of caregiver burden ratings, ${ }^{23}$ and our study suggests that patient mental health symptoms at the very EOL remain a significant predictor of caregiver burden. High caregiver burden is predictive of complicated grief after death, ${ }^{24}$ thus highlighting the need to address the psychological needs of patients at EOL to serve not only the patient but also the caregiver. When a patient suffers, the whole family suffers as well. Further research investigating strategies to reduce both patient psychological symptoms and caregiver burden are clearly warrant to provide comprehensive quality hospice care at EOL.

This study has a number of limitations, which are also limitations of the field of psychological symptom assessment at the very EOL in general. Using proxy interviews within two to three weeks of death to establish rates of psychological distress in the last week of life may have overestimated or underestimated patient's actual psychological distress. It also may have been a projection of the caregiver's own discomfort and not an accurate reflection of the patient's level of suffering. In addition, depressive symptoms were missing in $21 \%$ of the sample, whereas anxiety symptom data were missing in roughly $13 \%$. Caregivers often reported difficulty estimating psychological symptom burden in patients because of the patient's inability to communicate, for example, due to unconsciousness. In a similar study using hospice workers as proxies, hospice workers responded "do not know" for $26 \%$ of patients in regard to symptoms of worry, $22 \%$ for symptoms of sadness, and $17 \%$ for symptoms of nervousness, thus reflecting the difficulty of assessing psychological symptoms via proxy at the very EOL. ${ }^{6}$ We also did not access chart records to determine if hospice providers noted symptoms of anxiety and depression in the patients' medical 
records. It is thus unclear if the psychological symptoms reported by caregivers in our study represented overestimates or underestimates of the actual prevalence rates. Another limitation is the high refusal rate. Of the 1848 caregivers called, 804 (44\%) never answered the phone, 653 (35\%) declined to participate, whereas 391 (21\%) met eligibility criteria and enrolled in the parent study. An additional 40 (2\%) caregivers were not eligible for the present study because their care recipient had a live discharge from hospice. Participation in EOL research is a challenge, in general, given the sensitivity of the research topic and the vulnerability of the population of interest.

\section{Conclusion}

Effective psychological symptom management at the very EOL is essential to providing comprehensive hospice care. Our study revealed that caregivers of home hospice patients perceived moderate-to-severe psychological distress in just under half of patients in the last week of life, which is similar to results found in prior studies. ${ }^{5,6}$ Correlates of increased psychological symptom burden include caregiver burden (higher burden scores are associated with more psychological symptom distress) and patient age (younger patients are perceived to have more psychological distress). Given these prevalence rates, more research, both in regard to assessment and treatment, is needed to improve psychological symptoms at the very EOL to improve the quality of life for patients and their families throughout the full course of the illness trajectory.

\section{Disclosures and Acknowledgments}

The authors declared no potential conflict of interest with respect to the research, authorship, and/or publication of this article. Dr. Kozlov is supported by a fellowship from NIH/NIA (T32 AG049666-01A1, PI: M. C. R./H. P.). Dr. Phongtankuel's work is supported by a grant from the National Institute of Aging (R03 AG053284-01) and Weill Cornell Medical College Clinical and Translational Science Center. Dr. Reid is supported by grants from the National Institute on Aging (P30AG022845 and K24AGO53462). He is also the recipient of an investigatorinitiated grant from Pfizer Pharmaceuticals and the American Pain Society. Dr. Prigerson is supported by the several grants from the NIH (R35 CA197730 04; R21 CA218313; and T32 AG049666, PI: M. C. R./H. P.).

\section{References}

1. Mitchell AJ, Chan M, Bhatti H, et al. Prevalence of depression, anxiety, and adjustment disorder in oncological, haematological, and palliative-care settings: a meta-analysis of 94 interview-based studies. Lancet Oncol 2011;12:160-174. [PubMed: 21251875]

2. Stiefel F, Trill M, Berney A, Olarte J, Razavi D. Depression in palliative care: a pragmatic report from the expert working group of the European Association for Palliative Care. Support Care Cancer 2001;9(7):477-488. [PubMed: 11680829]

3. Cukor D, Ver Halen N, Fruchter Y. Anxiety and quality of life in ESRD. Semin Dial 2013;26:265268. [PubMed: 23432416]

4. Yohannes AM, Willgoss TG, Baldwin RC, Connolly MJ. Depression and anxiety in chronic heart failure and chronic obstructive pulmonary disease: prevalence, relevance, clinical implications and management principles. Int J Geriatr Psychiatry 2010;25:1209-1221. [PubMed: 20033905]

5. Garrigos A, Conill C, Saiz N, et al. Symptom prevalence in the last week of life. J Pain Symptom Manage 2002;14(6): 328-331.

6. Kutner JS, Kassner CT, Nowels DE. Symptom burden at the end of life: hospice providers' perceptions. J Pain Symptom Manage 2001;21(6):473-480. [PubMed: 11397605]

7. Wilson KG, Chochinov HM, Skirko MG, et al. Depression and anxiety disorders in palliative cancer care. J Pain Symptom Manage 2007;33(2):118-129. [PubMed: 17280918] 
8. Breitbart W, Rosenfeld B, Pessin H, et al. Depression, hopelessness, and desire for hastened death in terminally ill patients with cancer. JAMA 2000;284:2907. [PubMed: 11147988]

9. Patrick DL, Ferketich SL, Frame PS, et al. National Institutes of Health State-of-the-Science Conference Statement: symptom management in cancer: pain, depression, and fatigue, July 15-17, 2002. J Natl Cancer Inst 2003;95: 1110-1117. [PubMed: 12902440]

10. Lobchuk MM, Degner LF. Symptom experiences: perceptual accuracy between advanced-stage cancer patients and family caregivers in the home care setting. J Clin Oncol 2002;20:3495-3507. [PubMed: 12177111]

11. Facts and Figures Hospice care in America. Available from https://www.nhpco.org/sites/default/ files/public/Statistics_Research/2017_Facts_Figures.pdf. Accessed January 24, 2019.

12. Chow E, Wong R, Connolly R, et al. Prospective assessment of symptom palliation for patients attending a rapid response radiotherapy program: Feasibility of telephone follow-up. J Pain Symptom Manage 2001;22(2):649-656. [PubMed: 11495711]

13. de la Cruz M, Noguera A, San Miguel-Arregui MT, Williams J, Chisholm G, Bruera E. Delirium, agitation, and symptom distress within the final seven days of life among cancer patients receiving hospice care. Palliat Support Care 2015;13:211-216. [PubMed: 24556057]

14. Gilbertson-White S, Aouizerat BE, Jahan T, Miaskowski C. A review of the literature on multiple symptoms, their predictors, and associated outcomes in patients with advanced cancer. Palliat Support Care 2011;9(1): 81-102. [PubMed: 21352621]

15. Graessel E, Berth H, Lichte T, Grau H. Subjective care-giver burden: validity of the 10-item short version of the Burden Scale for Family Caregivers BSFC-s. BMC Geriatr 2014;14:23. [PubMed: 24555474]

16. Ringdal GI, Jordhøy MS, Kaasa S. Measuring Quality of Palliative Care: Psychometric Properties of the FAMCARE Scale Q. Available from https://link.springer.com/content/pdf/10.1023/A: 1022236430131.pdf. Accessed March 12, 2019.

17. Selby D, Cascella A, Gardiner K, et al. A Single Set of Numerical Cutpoints to Define moderate and severe symptoms for the Edmonton symptom assessment System. J Pain Symptom Manage 2010;39(2):241-249. [PubMed: 19963335]

18. Kroenke K, Spitzer RL, Williams JBW. The PHQ-9. J Gen Intern Med 2001;16:606-613. [PubMed: 11556941]

19. Spitzer RL, Kroenke K, Williams JBW, Löwe B. A brief measure for assessing generalized anxiety disorder: the GAD-7. Arch Intern Med 2006;166:1092-1097. [PubMed: 16717171]

20. Lorish CD, Maisiak R. The Face Scale: A Brief, Nonverbal Method for Assessing Patient Mood. Available from https://onlinelibrary.wiley.com/doi/pdf/10.1002/art.1780290714. Accessed March $1,2019$.

21. Brandt HE, Ooms ME, Deliens L, van der W, Ribbe MW. The last two days of life of nursing home patients - a nationwide study on causes of death and burdensome symptoms in The Netherlands. Palliat Med 2006;20:533-540. [PubMed: 16903407]

22. McKinley S, Stein-Parbury J, Chehelnabi A, Lovas J. Assessment of anxiety in intensive care patients by using the faces anxiety scale. Am J Crit Care 2004;13:146-152. [PubMed: 15043242]

23. Schrag A, Hovris A, Morley D, Quinn N, Jahanshahi M. Caregiver-burden in parkinson's disease is closely associated with psychiatric symptoms, falls, and disability. Parkinsonism Relat Disord 2006;12:35-41. [PubMed: 16271496]

24. Schulz R, Boerner K, Shear K, Zhang S, Gitlin LN. Predictors of complicated grief among dementia caregivers: a Prospective study of Bereavement. Am J Geriatr Psychiatry 2006;14:650658. [PubMed: 16861369] 

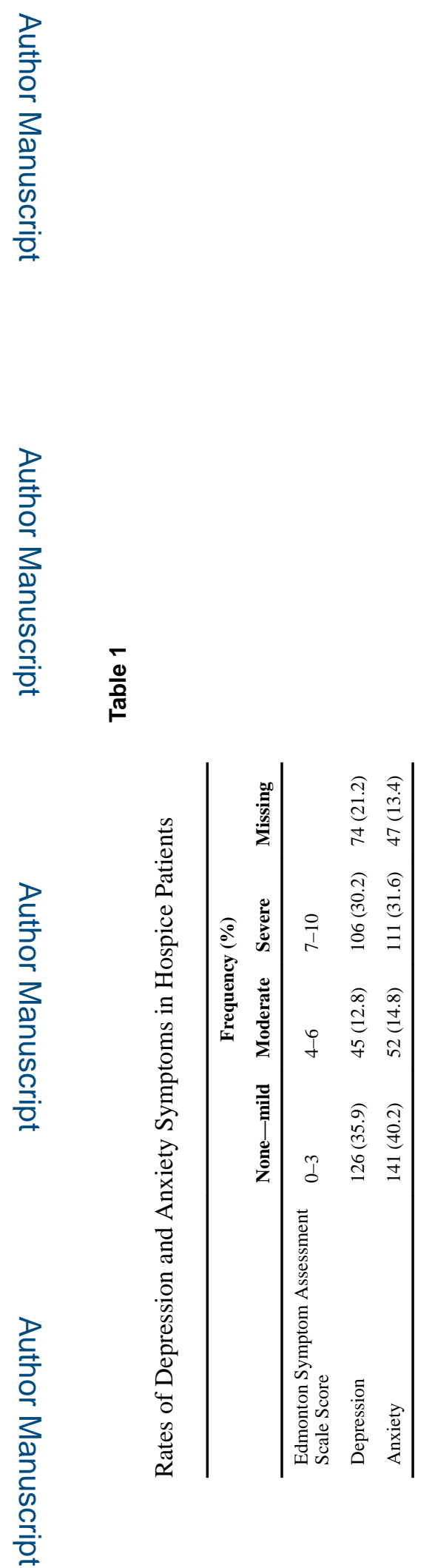


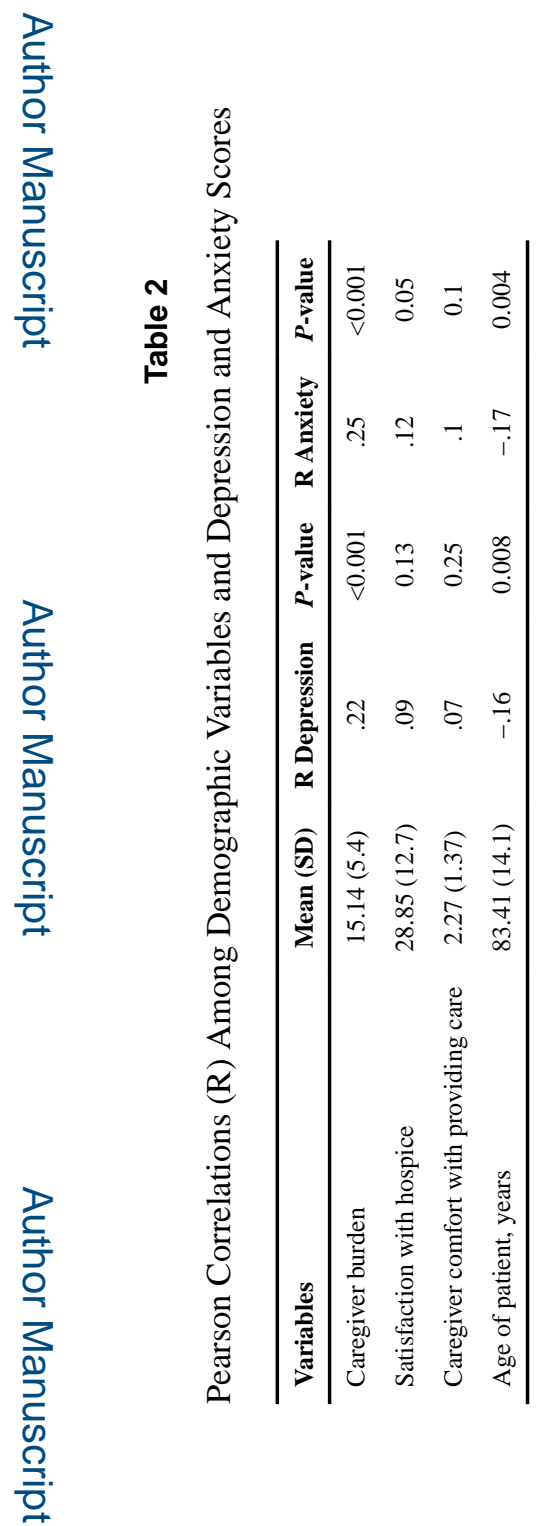




\section{로을}

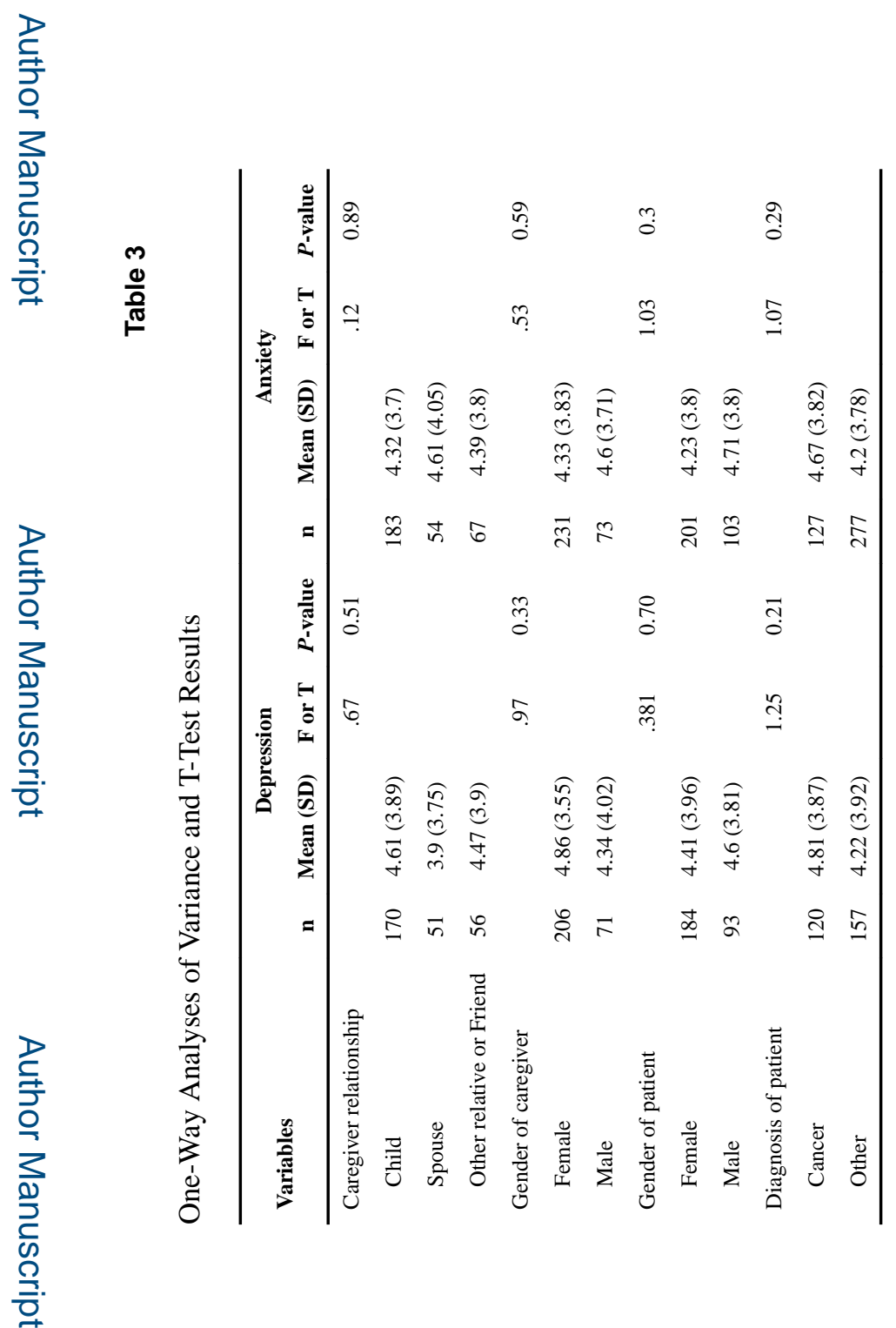

J Pain Symptom Manage. Author manuscript; available in PMC 2019 September 04. 